\title{
Risk factors of postoperative recurrence of primary spontaneous pneumothorax
}

\author{
Taiki Fujiwara^, Kazuhisa Tanaka, Takahide Toyoda, Terunaga Inage, Yuichi Sakairi, Fumihiro Ishibashi, \\ Hidemi Suzuki, Takahiro Nakajima, Ichiro Yoshino \\ Department of General Thoracic Surgery, Chiba University Graduate School of Medicine, Chiba, Japan \\ Contributions: (I) Conception and design: T Fujiwara; (II) Administrative support: I Yoshino; (III) Provision of study materials or patients: I Yoshino; \\ (IV) Collection and assembly of data: T Fujiwara, K Tanaka, T Toyoda, T Inage, Y Sakairi, F Ishibashi, H Suzuki, T Nakajima; (V) Data analysis and \\ interpretation: T Fujiwara, I Yoshino; (VI) Manuscript writing: All authors; (VII) Final approval of manuscript: All authors. \\ Correspondence to: Taiki Fujiwara, MD, PhD. Department of General Thoracic Surgery, Chiba University Graduate School of Medicine, 1-8-1 \\ Inohana, Chuo-ku, Chiba 260-8670, Japan. Email: taiki_ni_kiita@nifty.com.
}

Background: Spontaneous pneumothorax is common in relatively young, healthy patients. The risk factors for postoperative recurrence after correction are not fully understood.

Methods: We retrospectively reviewed the records of patients who underwent surgery for spontaneous pneumothorax between January 2006 and October 2017. We assessed the possible factors causing postoperative recurrence using univariate and multivariate analyses. We then used the inverse probability of treatment-weighted method to correct for confounding factors.

Results: Postoperative recurrence was observed in 41 of 233 patients (17.6\%). A significant association with recurrence was noted for primary spontaneous pneumothorax (PSP), never smokers, thoracoscopic surgery, patients younger than 30 years, operative time less than 100 minutes, and surgery by a resident surgeon. Patients younger than 30 years of age had a 5-year recurrence-free probability of $46.3 \%$. On multivariate analysis, patients younger than 30 years was an independent risk factor for recurrence. The confounding factors for recurrence of PSP included patients younger than 30 years, surgery by a resident surgeon, and thoracoscopic surgery. After adjusting for confounders, patients younger than 30 years was the only factor associated with recurrence $(\mathrm{P}=0.015)$. Patients who underwent bulla ligation with pleural reinforcement using an absorbable polyglycolic acid (PGA) sheet had a 4\% recurrence rate and a 5-year recurrence-free probability of $90.0 \%$.

Conclusions: Patients younger than 30 years of age is a significant factor for postoperative recurrence in patients with PSP. Surgeons may attempt to prevent postoperative recurrence by devising the operative method and pleural reinforcement methods.

Keywords: Primary spontaneous pneumothorax (PSP); postoperative recurrence; young patient; resident surgeon

Submitted Jul 14, 2020. Accepted for publication Sep 30, 2020.

doi: $10.21037 /$ jtd-20-2436

View this article at: http://dx.doi.org/10.21037/jtd-20-2436

\section{Introduction}

Primary spontaneous pneumothorax (PSP) is a common entity that develops in relatively young, healthy patients. Surgical management is indicated in patients with recurrent ipsilateral pneumothorax, simultaneous bilateral pneumothoraxes, and persistent air leakage $(1,2)$. Young patients with PSP represent an opportunity for surgical residents to train in thoracoscopic surgery. This is not the

^ ORCID: 0000-0002-6228-0808. 
case with secondary spontaneous pneumothorax (SSP), because surgery is sometimes complicated. Thoracoscopic surgery has been performed with increasing frequency in recent decades; however, the recurrence rate of pneumothorax after thoracoscopic surgery ranges from $2 \%$ to $14 \%$ - a higher rate than after thoracotomy $(3,4)$. To prevent postoperative recurrence, surgeons may perform additional procedures such as pleural reinforcement using oxidized regenerated cellulose (ORC) mesh or absorbable polyglycolic acid (PGA) sheets $(3,5-8)$. Young patients with PSP are reportedly at significant risk for postoperative recurrence $(3,9)$, possibly because of bulla neogenesis (3,8-11). The risk factors for postoperative recurrence of spontaneous pneumothorax are not fully understood. These factors, together with the phenomenon of bulla neogenesis, may contribute to the high rate of recurrence seen in young patients. We aimed to investigate the relation between postoperative recurrence and its risk factors. We present the following article in accordance with the STROBE reporting checklist (available at http://dx.doi.org/10.21037/jtd-202436).

\section{Methods}

\section{Study design}

We designed a retrospective study to review the records of patients who underwent surgery for spontaneous pneumothorax at Chiba University Hospital. This study was approved by the institutional review board of Chiba University Graduate School of Medicine, No.2996. The requirement for written consent was waived because of the retrospective nature of this study. Patients were notified of the study and given the option to optout. The study was conducted in accordance with the Declaration of Helsinki (as revised in 2013). We included patients who underwent conservative treatment for recurrent pneumothorax, such as indwelling chest-tube drainage, and patients who had persistent air leakage. We excluded patients with secondary ipsilateral recurrent pneumothorax after previous surgery.

\section{Surgery for spontaneous pneumothorax}

A double-lumen endotracheal tube was inserted into the airway under general anesthesia, and surgery was performed during selective ventilation of the contralateral lung. Surgery was carried out with patients in the lateral position. The first choice for intervention at our institution was thoracoscopic surgery using a $5-\mathrm{mm}, 30^{\circ}$ video thoracoscope and 3 intercostal ports $(5-12 \mathrm{~mm})$ for access. The working ports were typically placed in the third intercostal space in the anterior and posterior axillary line, and the fifth intercostal space in the midaxillary line, but could be placed elsewhere depending on the requirements of the case. Thoracotomy (approximately 4-8 cm) was an option when pleural adhesions prevented the safe use of thoracoscopy or port placement was not suitable for procedures such as pulmorrhaphy. Surgeons were classified as residents, fellows, and specialists. Since our hospital is an educational facility, residents or fellows are selected for procedures that are not expected to be difficult; their procedures are conducted under the guidance of specialists. Bullectomy or bulla ligation were performed to halt air leakage from the pulmonary parenchyma; in some patients, pleural cautery or pulmorrhaphy was also performed. The choice of surgical procedure was made by the surgeons after examining the preoperative CT findings and the immediate intraoperative findings. When bullae were numerous or their stalks were large, we opted for bullectomy. When bullae were small and few in number or their stalks were small, we performed bulla ligation. We performed pleural cautery when the origin of air leakage was not clear but the location was thought to be at the site of the inflammatory changes. We performed pulmorrhaphy when bullectomy or bulla ligation were deemed difficultfor example, when the site of air leakage was near the hilum or when emphysematous changes were extremely prevalent. Pleural reinforcement was considered an optional procedure to prevent recurrence; this was left to the surgeons' discretion. All patients were followed for at least 1 month postoperatively on an outpatient basis. All patients were instructed to consult the follow-up clinic when any symptoms occurred that were suspicious for recurrent pneumothorax.

\section{Review of data for patients with spontaneous pneumothorax}

We reviewed the medical records of each patient to obtain clinical and perioperative information, including patient age, sex, type of spontaneous pneumothorax (primary or secondary), smoking habits, laterality of the pneumothorax, history of ipsilateral and contralateral spontaneous pneumothorax before surgery, surgical approach (thoracoscopic surgery or thoracotomy), surgical methods (bullectomy using an endoscopic stapler or bulla ligation 
Table 1 Characteristics of the enrolled patients with spontaneous pneumothorax

\begin{tabular}{|c|c|}
\hline Patients & Total $n=233$ \\
\hline \multicolumn{2}{|l|}{ Sex } \\
\hline Men/women & $186 / 47$ \\
\hline \multicolumn{2}{|l|}{ Type of SP } \\
\hline Primary/secondary & $129 / 104$ \\
\hline \multicolumn{2}{|l|}{ Smoking habits } \\
\hline Ever/never/unknown & $111 / 113 / 9$ \\
\hline \multicolumn{2}{|l|}{ Laterality } \\
\hline Left/right/bilateral & $119 / 113 / 1$ \\
\hline \multicolumn{2}{|l|}{ History of ipsilateral SP } \\
\hline Yes/no & $127 / 106$ \\
\hline \multicolumn{2}{|l|}{ History of contralateral SP } \\
\hline Yes/no & $173 / 60$ \\
\hline \multicolumn{2}{|l|}{ Surgical approach } \\
\hline Thoracoscopic surgery/thoracotomy & $181 / 52$ \\
\hline \multicolumn{2}{|l|}{ Surgical methods } \\
\hline Bullectomy/bulla ligation/other & $150 / 61 / 22$ \\
\hline \multicolumn{2}{|l|}{ Pleural reinforcement } \\
\hline ORC/PGA/fibrin glue/other/none & $125 / 80 / 66 / 22 / 20$ \\
\hline \multicolumn{2}{|l|}{ Operative time } \\
\hline$<100>100 \mathrm{~min}$ & $119 / 114$ \\
\hline \multicolumn{2}{|l|}{ Surgeon } \\
\hline Resident/fellow/specialist & $65 / 92 / 76$ \\
\hline \multicolumn{2}{|l|}{ Number of cases by age, $\mathrm{n}(\%)$} \\
\hline$<20$ years & $53(22.7)$ \\
\hline$<30$ years & $65(27.9)$ \\
\hline$<40$ years & $17(7.3)$ \\
\hline$<50$ years & $26(11.2)$ \\
\hline$<60$ years & $10(4.3)$ \\
\hline$<70$ years & $25(10.7)$ \\
\hline$<80$ years & $32(13.7)$ \\
\hline$<90$ years & $5(2.1)$ \\
\hline
\end{tabular}

ORC, oxidized regenerated cellulose mesh; PGA, absorbable polyglycol acid sheets; SP, Spontaneous pneumothorax. using a pre-tied loop ligature), pleural reinforcement (ORC mesh, absorbable PGA sheets, or fibrin glue), operative time $(<100$ or $>100 \mathrm{~min})$, and the type of surgeon. We classified surgeons with less than 3 years of experience as residents, and those with more than 3 years of experience who were training as specialists in general thoracic surgeons as fellows. Specialists were general thoracic surgeons certified by The Japanese Board of General Thoracic Surgery. Postoperative recurrence was defined as ipsilateral pneumothorax after surgery.

\section{Statistical analyses}

We used recurrence-free probability (RFP), multivariate analysis, and the inverse probability of treatment-weighted (IPTW) method (that employs the propensity score) to assess the relation between postoperative recurrence of pneumothorax and the risk factors in question. We calculated RFP using the Kaplan-Meier method. The Cox regression analysis was used for multivariate analysis. Finally, we performed our propensity score analysis by Cox regression analysis using the IPTW method. A P value of $<0.05$ indicated statistical significance. The hazard ratio (HR) and $95 \%$ confidence intervals $(95 \% \mathrm{CI})$ were calculated for each variable. We used the EZR software package (http://www.jichi.ac.jp/saitama-sct/SaitamaHP.files/ statmed.html) to perform all statistical analyses (12).

\section{Results}

\section{Patient profile}

Between January 2006 and October 2017, a total of 233 patients with spontaneous pneumothorax underwent surgery for this indication at Chiba University Hospital. Patient characteristics are summarized in Table 1. There were 186 men and 47 women, with an average age of 39.0 years. A total of 53 patients were younger than 20 years of age $(22.7 \%)$, and 65 were between 20 and 29 years of age (27.9\%). Of patients with PSP, $84.5 \%$ were younger than 30 years of age (Table S1). A total of 129 patients (55.4\%) had PSP, and 113 patients (48.5\%) were never-smokers. The type of spontaneous pneumothorax was categorized by comprehensively evaluating the preoperative CT findings, any history of pulmonary disease, and the patient's smoking history. The underlying diseases in patients with SSP included chronic obstructive pulmonary disease, interstitial pneumonia, and Birt-Hogg-Dubé syndrome (Table S2). 
Table 2 Postoperative recurrence and recurrence-free probability with spontaneous pneumothorax

\begin{tabular}{lcc}
\hline & Recurrence (rate, \%) & 5-year RFP, \% \\
\hline Recurrence & & \\
Yes & $41(17.6)$ & 65.6 \\
No & $192(82.4)$ & - \\
Recurrence by age & & 44.2 \\
$<20$ years & $17(32.1)$ & 48.5 \\
$<30$ years & $17(26.2)$ & 100.0 \\
$<40$ years & $0(0.0)$ & 62.8 \\
$<50$ years & $4(15.4)$ & 63.9 \\
$<60$ years & $1(10.0)$ & 95.2 \\
$<70$ years & $1(4.0)$ & 62.2 \\
$<80$ years & $1(3.1)$ & 100.0 \\
$<90$ years & $0(0.0)$ & \\
\hline
\end{tabular}

RFP, recurrence-free probability.

Thoracoscopic surgery was the most common approach, performed in 181 patients $(77.7 \%)$ overall and in $90.6 \%$ of patients with PSP (Table S1). A total of 150 patients (64.4\%) underwent bullectomy, and 61 patients (26.2\%) underwent bulla ligation. Pleural reinforcement to prevent recurrence was performed in almost all patients (91.4\%): ORC mesh was the most commonly used material [125 patients (53.6\%)], but absorbable PGA sheets [80 patients $(34.3 \%)$ ] and fibrin glue [66 patients $(28.3 \%)$ ] were also used. The median operative time was 96 minutes. A total of 65 patients (27.9\%) underwent surgery by a resident $[41.4 \%$ of patients with PSP had a resident as their primary surgeon (Table S1)], 92 (39.5\%) underwent surgery by a fellow, and $76(32.6 \%)$ underwent surgery by a specialist. Additional treatment for prolonged air leakage, such as chemical pleurodesis, was performed in 30 patients.

A total of 41 patients (17.6\%) experienced recurrence (Table 2). Of these, 17 (32.1\%) were younger than 20 years of age and $17(26.2 \%)$ were between 20 and 29 years of age. Of the 84 patients younger than 30 years of age, 34 (28.8\%) experienced a recurrence, accounting for $82.9 \%$ of the 41 patients with recurrence.

The 5 -year RFP was $65.6 \%$ for all patients (Table 3). In patients younger than 20 years of age, the RFP was $44.2 \%$, and in those between 20 and 29 years of age it was $48.5 \%$.

The recurrence rate for bullectomy was $20.7 \%$ (31/150 patients), and the recurrence rate was $16.4 \%(10 / 61$ patients) for bulla ligation $(\mathrm{P}=0.5669$, Table $\mathrm{S} 3)$. The 5 -year RFP for patients undergoing bullectomy was $64.5 \%$, and in patients undergoing bulla ligation it was $61.1 \%$ $(\mathrm{P}=0.181$; Table 3). The recurrence rate for patients who underwent pleural reinforcement with ORC mesh was $20.8 \%$, and the rate for those who received absorbable PGA sheets was $8.6 \%(\mathrm{P}=0.02$; Table $\mathrm{S} 3)$. The 5 -year RFP for patients who underwent pleural reinforcement using ORC mesh was $56.7 \%(\mathrm{P}=0.281)$, and for those who underwent reinforcement with absorbable PGA sheets it was $86.4 \%$ $(\mathrm{P}=0.261$; Table 4). The recurrence rate for ligation with reinforcement using a PGA sheet was $4.0 \%$ (1/25 patients; $\mathrm{P}=0.09)$ and the 5 -year RFP was $90.0 \%(\mathrm{P}=0.459)$

\section{Recurrence-free probability and multivariate analysis of risk factors for recurrence}

Table 4 shows that PSP $(\mathrm{P}<0.001)$, never-smoker status $(\mathrm{P}<0.001)$, left-sided pneumothorax $(\mathrm{P}=0.049)$, thoracoscopic surgery $(\mathrm{P}=0.008)$, operative time less than $100 \mathrm{~min}(\mathrm{P}<0.001)$, surgery by a resident surgeon $(\mathrm{P}=0.008)$, and younger than 30 years old $(\mathrm{P}<0.001)$ were significant risk factors for postoperative recurrence. Factors for multivariate analysis were selected based on the results of RFP analysis. Multivariate analysis (Table 4) indicated that age younger than 30 years (HR 6.54; 95\% CI, 1.34-32.1; $\mathrm{P}=0.021)$ was an independent predictor of recurrence.

\section{Assessment of risk factors for recurrence using the IPTW method}

According to our univariate analysis, the confounding factors for PSP were the patient's smoking habit, the laterality of the pneumothorax, the surgical method, the surgical approach, the classification of surgeon, the performance of pleural reinforcement, and patient age younger than 30 years (Table S1). When IPTW analysis was performed to discriminate significant confounders, only patient age less than 30 years was revealed to be a significant factor (HR 14.3; 95 CI, 1.21-783; $\mathrm{P}=0.0153$; Table 4).

\section{Discussion}

For the patients with recurrent spontaneous pneumothorax, thoracoscopic bullectomy is considered a definitive treatment choice $(3,4)$. Despite its high recurrence rate when performed alone (16.3-24.1\%) (9,13-15). Various additional intraoperative procedures were designed to be 
Table 3 Clinical characteristics and recurrence-free probability for postoperative recurrence of spontaneous pneumothorax

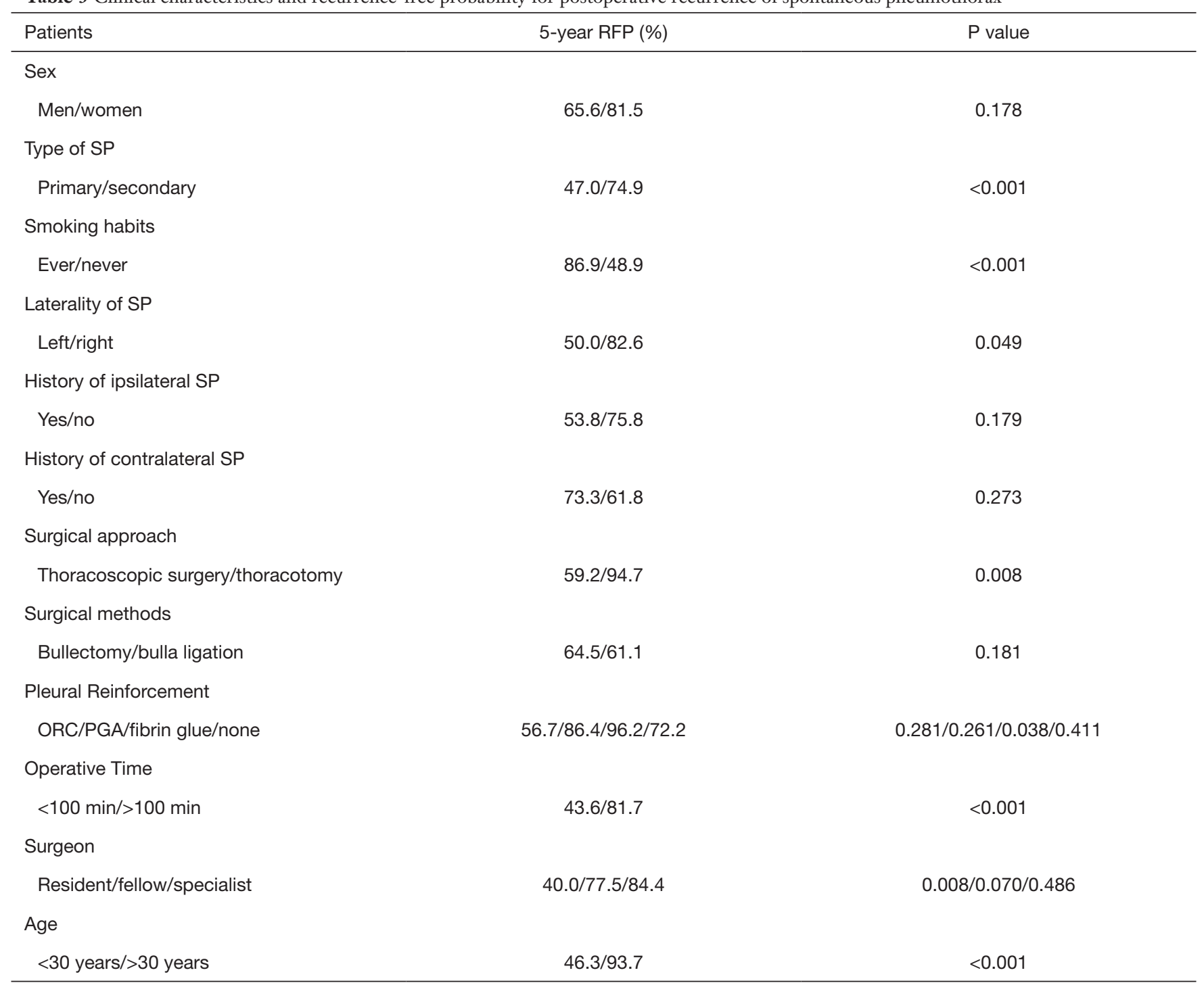

RFP, recurrence-free probability; ORC, oxidized regenerated cellulose mesh; PGA, absorbable polyglycol acid sheets; SP, spontaneous pneumothorax.

performed for preventing postoperative recurrence-pleural reinforcement is among the more effective procedures. Cho et al. (16) reported a $4.8 \%$ recurrence rate using absorbable ORC mesh coverage and fibrin glue in patients with PSP, while Lee et al. (17) reported a 3.9\% recurrence rate using absorbable PGA sheets to widely cover the staple line and pleural abrasion in patients with PSP.

Postoperative recurrence of spontaneous pneumothorax especially in young patients is relatively common due to bulla neogenesis (9). Nakayama et al. reported that after performing thoracoscopic bullectomy for PSP with application of an absorbable PGA sheet, patients younger than 23 years of age have a lower probability of 5 -year recurrence-free status than those aged 23 years or older (78.1\% vs. 93.8\%) (3). The recurrence rate in our study was higher $(28.8 \%)$ and 5 - year RFP was lower (46.3\%) in patients younger than 30 years of age, even with pleural reinforcement.

The treatment of young patients with PSP presents opportunities for residents to train in thoracoscopic surgery. Since our hospital is an educational facility, residents or fellows perform surgery, under the guidance of specialists, 
Table 4 Multivariate analysis and the IPTW method using the propensity score for postoperative recurrence with spontaneous pneumothorax

\begin{tabular}{|c|c|c|c|c|c|c|}
\hline Factors & \multicolumn{3}{|c|}{ Multivariate analysis } & \multicolumn{3}{|c|}{ IPTW method } \\
\hline Type of SP: secondary & 0.366 & $0.081-0.65$ & 0.190 & 0.640 & $0.048-6.24$ & $>0.99$ \\
\hline Smoking habits: ever & 0.507 & $0.197-1.31$ & 0.161 & 0.163 & $0.003-1.85$ & 0.149 \\
\hline Laterality of SP: left & 1.09 & $0.508-2.34$ & 0.822 & 1.91 & $0.196-25.3$ & 0.646 \\
\hline Surgeon: resident & 1.52 & $0.693-3.35$ & 0.294 & 4.78 & $0.472-65.7$ & 0.116 \\
\hline Operative time: $>100 \mathrm{~min}$ & 0.706 & $0.323-1.54$ & 0.382 & 0.707 & $0.054-6.90$ & $>0.99$ \\
\hline Age: $<30$ years & 6.54 & $1.340-32.1$ & 0.021 & 14.3 & $1.21-783$ & 0.015 \\
\hline
\end{tabular}

HR, hazard ratio; IPTW, inverse probability of treatment-weighted; SP, spontaneous pneumothorax.

if the procedure is not expected to be difficult. In contrast to SSP, surgery for PSP in our study often involved resident surgeons. We therefore speculate that the higher rate of recurrence in surgery for PSP may be due to surgeon inexperience. To eliminate this as a possible confounding factor, we used propensity scoring to determine that patient younger than 30 years of age is the only significant factor for postoperative recurrence, regardless of the type of surgeon or surgical approach.

Our study demonstrates a trend toward a higher recurrence rate for bullectomy (5-year RFP: $64.5 \%$ ) than for bulla ligation (5-year RFP: $61.1 \% ; \mathrm{P}=0.181$ ). Our results are consistent with those of Fujino et al., who report that the recurrence rate for bulla ligation in patients with spontaneous pneumothorax is significantly lower than that of bullectomy (5.8\% vs. $17.8 \%$ ) (18). We speculate that, of the various surgical methods, bulla ligation is less stressful on the surgical site than bullectomy, and as a result, bulla neogenesis is less likely. Our study also showed that the postoperative recurrence rate is higher in patients who undergo reinforcement using ORC mesh than with PGA sheets, with rates similar to those found by Ozawa $e t$ al. (22.8\% vs. 3.6\%) (8). Pleural reinforcement using ORC sheets prevents recurrence by thickening the visceral pleura near the surgical site. It also prevents adhesion formation between the surgical site and the chest wall. However, the mesh sheet may fall into the chest cavity. We speculate that this may cause recurrence more frequently than with the use of PGA sheets. Although we can only report a limited number of cases, we found that ligation with reinforcement using a PGA sheet may be an effective surgical method for preventing recurrence, with only a $4 \%$ recurrence rate and a 5 -year RFP of $90.0 \%$. It is very difficult to determine exactly why bulla ligation with the use of PGA sheets causes less recurrence, but it may be better for surgeons to combine methods that are less likely to lead to recurrence. Surgeons should think carefully about the surgical and pleural reinforcement methods used for each patient. One method of reducing postoperative recurrence is the use of $50 \%$ glucose solution for pleural reinforcement, as described by Fujino et al. (19). Abrasion of the surrounding pleural lesion after bullectomy or bulla ligation may also reduce recurrence.

The major limitations of this study were that it was conducted at a single institution, and the results were based on a small number of patients. Multicenter prospective trials are needed to confirm the results of this study.

\section{Conclusions}

In conclusion, patients younger than 30 years old with spontaneous pneumothorax, especially PSP, had a significantly higher recurrence rate after surgical repair. It is necessary to prevent postoperative recurrence by devising the operative method and pleural reinforcement method.

\section{Acknowledgments}

This data was presented at $35^{\text {th }}$ Annual Meeting of the Japanese Association for Chest Surgery, Chiba, Japan, May 2018.

Funding: None. 


\section{Footnote}

Reporting Checklist: The authors have completed the STROBE reporting Checklist. Available at http://dx.doi. org/10.21037/jtd-20-2436

Data Sharing Statement: Available at http://dx.doi. org/10.21037/jtd-20-2436

Peer Review File: Available at http://dx.doi.org/10.21037/jtd20-2436

Conflicts of Interest: All authors have completed the ICMJE uniform disclosure form (available at http://dx.doi. org/10.21037/jtd-20-2436). YS serves as an unpaid editorial board member of Fournal of Thoracic Disease from Apr 2019 to Mar 2021. The authors have no other conflicts of interest to declare.

Ethical Statement: The authors are accountable for all aspects of the work in ensuring that questions related to the accuracy or integrity of any part of the work are appropriately investigated and resolved. This study was approved by the institutional review board of Chiba University Graduate School of Medicine, No.2996. The requirement for written consent was waived because of the retrospective nature of this study. The study was conducted in accordance with the Declaration of Helsinki (as revised in 2013).

Open Access Statement: This is an Open Access article distributed in accordance with the Creative Commons Attribution-NonCommercial-NoDerivs 4.0 International License (CC BY-NC-ND 4.0), which permits the noncommercial replication and distribution of the article with the strict proviso that no changes or edits are made and the original work is properly cited (including links to both the formal publication through the relevant DOI and the license). See: https://creativecommons.org/licenses/by-nc-nd/4.0/.

\section{References}

1. Onuki T, Kawamura T, Kawabata S, et al. Neo-generation of neogenetic bullae after surgery for spontaneous pneumothorax in young adults: a prospective study. J Cardiothorac Surg 2019;14:20

2. MacDuff A, Arnold A, Harvey J, et al. Management of spontaneous pneumothorax: British Thoracic Society
Pleural Disease Guideline 2010. Thorax 2010;65 Suppl 2:ii18-ii31.

3. Nakayama T, Takahashi $Y$, Uehara H, et al. Outcome and risk factors of recurrence after thoracoscopic bullectomy in young adults with primary spontaneous pneumothorax. Surg Today 2017;47:859-64.

4. Sahn SA, Heffner JE. Spontaneous pneumothorax. N Engl J Med 2000;342:868-74.

5. Muramatsu T, Nishii T, Takeshita S, et al. Preventing recurrence of spontaneous pneumothorax after thoracoscopic surgery: a review of recent results. Surg Today 2010;40:696-9.

6. Sakamoto K, Takei H, Nishii T, et al. Staple line coverage with absorbable mesh after thoracoscopic bullectomy for spontaneous pneumothorax. Surg Endosc 2004;18:478-81.

7. Lee S, Kim HR, Cho S, et al. Korean Pneumothorax Study Group. Staple line coverage after bullectomy for primary spontaneous pneumothorax: a randomized trial. Ann Thorac Surg 2014;98:2005-11.

8. Ozawa Y, Sakai M, Ichimura H. Covering the staple line with polyglycolic acid sheet versus oxidized regenerated cellulose mesh after thoracoscopic bullectomy for primary spontaneous pneumothorax. Gen Thorac Cardiovasc Surg 2018;66:419-24

9. Tsuboshima K, Matoba Y, Wakahara T, et al. Natural history of bulla neogenesis for primary spontaneous pneumothorax: a propensity score analysis. Gen Thorac Cardiovasc Surg 2019;67:464-9.

10. Huang H, Ji H, Tian H. Risk factors for recurrence of primary spontaneous pneumothorax after thoracoscopic surgery. Biosci Trends 2015;9:193-7.

11. Noh D, Lee S, Haam SJ, et al. Recurrence of primary spontaneous pneumothorax in young adults and children. Interact Cardiovasc Thorac Surg 2015;21:195-9.

12. Kanda Y. Investigation of the freely available easy-touse software 'EZR' for medical statistics. Bone Marrow Transplantation 2013;48:452-8.

13. Hirai K, Kawashima T, Takeuchi S, et al. Covering the staple line with a polyglycolic acid sheet after bullectomy for primary spontaneous pneumothorax prevents postoperative recurrent pneumothorax. J Thorac Dis 2015;7:1978-85.

14. Cardillo G, Bintcliffe OJ, Carleo F, et al. Primary spontaneous pneumothorax: a cohort study of VATS with talc poudrage. Thorax 2016;71:847-53.

15. Hong KP, Kim DK, Kang KH. Staple line coverage with a polyglycolic acid patch and fibrin glue without pleural 
abrasion after thoracoscopic bullectomy for primary spontaneous pneumothorax. Korean J Thorac Cardiovasc Surg 2016;49:85-91.

16. Cho S, Huh DM, Kim BH, et al. Staple line covering procedure after thoracoscopic bullectomy for the management of primary spontaneous pneumothorax. Thorac Cardiovasc Surg 2008;56:217-20.

17. Lee S, Park SY, Bae MK, et al. Efficacy of polyglycolic acid sheet after thoracoscopic bullectomy for spontaneous

Cite this article as: Fujiwara T, Tanaka K, Toyoda T, Inage T, Sakairi Y, Ishibashi F, Suzuki H, Nakajima T, Yoshino I. Risk factors of postoperative recurrence of primary spontaneous pneumothorax. J Thorac Dis 2020;12(11):6458-6465. doi: $10.21037 /$ jtd-20-2436 pneumothorax. Ann Thorac Surg 2013;95:1919-23.

18. Fujino M, Yamakawa H, Matsui Y, et al. Evaluation of video-assisted thoracoscopic surgery for double loop ligation of the bullae for spontaneous pneumothorax. JPN J Chest Surg 2010;24:999-1003.

19. Fujino K, Motooka Y, Koga T, et al. Novel approach to pleurodesis with $50 \%$ glucose for air leakage after lung resection or pneumothorax. Surg Today 2016;46:599-602. 